ORIGINAL

\title{
Dinámica de impregnación al vacío en apio (Apium graveolens L.) y pepino (Cucumis sativus L.)
}

\section{Vacuum impregnation dynamic on celery (Apium graveolens L.) and cucumber (Cucumis sativus L.)}

\author{
Yisell Martelo C, ${ }^{1 *}$ M.Sc, Misael Cortés ${ }^{\prime}{ }^{1}$ Ph.D, Diego Restrepo $M,{ }^{1}$ M.Sc.
}

${ }^{1}$ Universidad Nacional de Colombia, Facultad de Ciencias Agropecuarias, Departamento de Ingeniería Agrícola y de Alimentos, Medellín, Colombia. *Correspondencia: mcortesro@unal.edu.co.

Recibido: Agosto de 2009; Aceptado: Enero de 2011.

\section{RESUMEN}

Objetivo. Evaluar la respuesta a la impregnación al vacio (IV) en apio y pepino, con soluciones isotónicas de $\mathrm{NaCl}$. Materiales y métodos. Se determinaron variables de impregnación en troncos de apio y rodajas de pepino ( 3 posiciones diferentes a lo largo de su estructura), considerando, fracción y deformación volumétrica en la etapa de vacío ( $X_{1}$ y $\gamma 1$ ) y atmosférica ( $X$ y $\gamma$ ), y la porosidad disponible (Ee) al proceso IV. Resultados. El apio y pepino no presentaron diferencias estadísticas por efecto de la posición. En las etapas de proceso se obtuvieron para el apio y el pepino valores de X1 $(-14.32 \pm 2.75$ y $-5.51 \pm 1.76 \%, \gamma 1(-0.587 \pm 0.69$ y $-0.079 \pm 0.99 \%), X(13.49 \pm 2.32$ y $6.72 \pm 2.72 \%), \gamma(-1.40 \pm 1.042 \%$ y $-2.33 \pm 1.26 \%)$ y Ee $(15.73 \pm 2.31$ y $9.35 \pm 2.57 \%)$, respectivamente. Estos resultados indicaron una salida de líquido nativo $\left(\mathrm{X}_{1}<0\right)$ y una ligera contracción volumétrica de las estructuras ( $\gamma$ y $\gamma 1<0$ ), lo cual se evidenció microestructuralmente. Conclusiones. La respuesta a la IV en apio y pepino, permite identificar estas matrices alimentarias, como aptas para la incorporación de componentes que le proporcionen un valor agregado a estos productos.

Palabras clave: Apium graveolens, Cucumis sativus, hortalizas, procesamiento de alimentos. (Fuente: AIMS). 


\section{ABSTRACT}

Objective. To evaluate the response to vacuum impregnation (IV) in celery and cucumber, with isotonic $\mathrm{NaCl}$. Materials and methods. Impregnation variables were determined in celery sticks and cucumber slices (3 different positions along their structure), considering, fraction and volumetric deformation in the vacuum stage $\left(X_{1}\right.$ and $\left.\gamma 1\right)$ and atmospheric $(X$ and $\gamma$ ), and the available porosity to the process IV (Ee). Results. Celery and cucumber were not statistically different for effect of the position. In the stages process were obtained for the celery and the cucumber values of $X_{1}(-14.32 \pm 2.75$ and $-5.51 \pm 1.76 \%, \gamma 1(-0.587 \pm 0.69$ and $-0.079 \pm 0.99 \%), X(13.49 \pm 2.32$ and $6.72 \pm 2.72 \%),(-1.40 \pm 1.042 \%$ and $-2.33 \pm 1.26 \%)$ and $\mathrm{Ee}(15.73 \pm 2.31$ and $9.35 \pm 2.57 \%)$, respectively. These results indicated a native liquid output $(X 1<0)$ and a slight volumetric contraction of the structures $(\gamma$ and $\gamma 1<0)$, which was evidenced microstructurally. Conclusions. The dynamics of IV in celery and cucumber, to identify those food matrices, as suitable for the incorporation of components that provide a value added to these products.

Key words: Apium graveolens, Cucumis sativus, food processing, vegetables. (Source: AIMS).

\section{INTRODUCCIÓN}

Actualmente existe una considerable demanda en países desarrollados y subdesarrollados, de productos vegetales por el importante papel que estos cumplen en la dieta humana. En este sentido vegetales y hortalizas troceadas, empacadas y refrigeradas (productos mínimamente procesados), podrían ofrecer en el presente y futuro, una interesante incursión de nuevas líneas de alimentos, modificados en su estructura y composición, adicionados con nutrientes, antioxidantes, antimicrobianos, crioprotectores, entre otros, con el objeto de mejorar su calidad y extender su vida útil.

La técnica de impregnación al vacío (IV) hace posible la incorporación de sustancias disueltas, emulsificadas o en suspensión directamente dentro de estructuras porosas, de una forma controlada, permitiendo la obtención de rápidos cambios composicionales y estructurales en las matrices que así lo permiten, como es el caso de frutas, hortalizas, tubérculos, entre otros, las cuales poseen características porosas en su estructura, permiten el proceso de incorporación de componentes mejoradores de textura, sabor, color o de componentes fisiológicamente activos (CFA) $(1,2)$. Diferentes aplicaciones de la técnica
IV han sido realizados en el campo de los alimentos (3-6), donde la mayoría de las publicaciones se relacionan principalmente con frutas (7-12) y pocas en hortalizas (1317). El modelo matemático para el proceso IV ha sido desarrollado en términos de la respuesta del tejido de la planta al proceso IV: fracción y deformación volumétrica de impregnación, tanto en la etapa de vacío $\left(\mathrm{X}_{1}: \mathrm{m}^{3}\right.$ de solución impregnada $/ \mathrm{m}^{3}$ de muestra inicial y $\gamma 1 \mathrm{~m}^{3}$ de deformación en la muestra $/ \mathrm{m}^{3}$ de muestra inicial), así como en el proceso global con el restablecimiento de la presión atmosférica $(X, \gamma)$ y la porosidad eficaz o disponible (Ee: $\mathrm{m}^{3}$ de gas $/ \mathrm{m}^{3}$ de muestra), el cual describe el acoplamiento del mecanismo hidrodinámico (MHD) y el fenómeno deformación-relajación (FDR) de las matrices alimentarias porosas cuando son inmersas en líquidos al ser sometidos a cambios de presión (18). Estos parámetros de impregnación dependen de las condiciones del proceso, de propiedades mecánicas y estructurales del tejido, así como de la viscosidad del líquido de impregnación (1).

Desde el punto de vista de anatomía microscópica el apio presenta en su mayoría células colenquimáticas con paredes celulares mucho más gruesas y 
normalmente largas y empaquetadas en fibras, a modo de cuerdas comunes en regiones subepidérmicas de los tallos; y el pepino es un fruto con numerosas células parenquimatosas. Estos vegetales son conocidos como los de menor valor energético, ocupando el apio el segundo lugar después del pepino, con un aporte de 14 y $20 \mathrm{kcal}$ respectivamente. El apio es un vegetal cuyas partes son todas comestibles, con contenidos de vitamina $C$ y vitamina $A$ de 32.0 y $0.207 \mathrm{mg} / 100 \mathrm{~g}$ respectivamente, y rico en potasio (280.00 mg) (19). Para pepinos frescos, el contenido de agua es muy alto (96\%), con un aporte de $4.7 \mathrm{mg}$ de ácido ascórbico, $14 \mathrm{mg}$ calcio, $149 \mathrm{mg}$ potasio, 17 $\mathrm{mg}$ de fósforo y $11 \mathrm{mg}$ magnesio por $100 \mathrm{~g}$ de producto fresco (20). Comparado con el promedio de las hortalizas, el contenido en vitamina C, A, tiamina y fibra es menor (19).

Debido al gran consumo de estos alimentos en la elaboración de ensaladas, se genera un interés industrial por la facilidad de adaptación de estos al procesamiento IV $(15,16)$. El objetivo de este trabajo fue evaluar la respuesta a la aplicación de la técnica IV en apio y pepino, con soluciones isotónicas de $\mathrm{NaCl}$.

\section{MATERIALES Y MÉTODOS}

Materias primas. Se utilizaron apio criollo (Apium graveolens $L$.var. dulce) y pepino cohombro (Cucumis sativus L.), obtenidos de un mercado local, sin defectos o daños causados por plagas, de coloración uniforme; los pepinos con longitudes entre 20 y $30 \mathrm{~cm}$. Las formas experimentales para el apio fueron de un grosor (J) entre 3-4 cm., espesor del tallo (K) entre 1 y $1.5 \mathrm{~cm}$. y altura $(\mathrm{H})$ de $2 \mathrm{~cm}$. Se consideraron 3 posiciones a lo largo del pecíolo, posición 1: zona lado cercano a las hojas, posición 2: zona media, posición 3: zona cercana al cuello de la raíz. Para el pepino las formas consistieron en rodajas cilíndricas del tejido del mesocarpio, sin semillas, de $1 \mathrm{~cm}$ de espesor (F) y diámetros interno $\left(\Phi_{\text {int }}\right) \approx 2.5-3.0 \mathrm{~cm}$, y externo $\left(\Phi_{\text {ext }}\right) \approx 3-5 \mathrm{~cm}$. Se consideraron 3 posiciones, posición 1: extremo lado ápice, posición 2: zona media, posición 3: extremo contrario al lado ápice. Las posiciones 1 y 3 se fijaron aproximadamente a $8.5 \mathrm{~cm}$ de los extremos. La figura 1 ilustra las formas correspondientes de cada hortaliza.

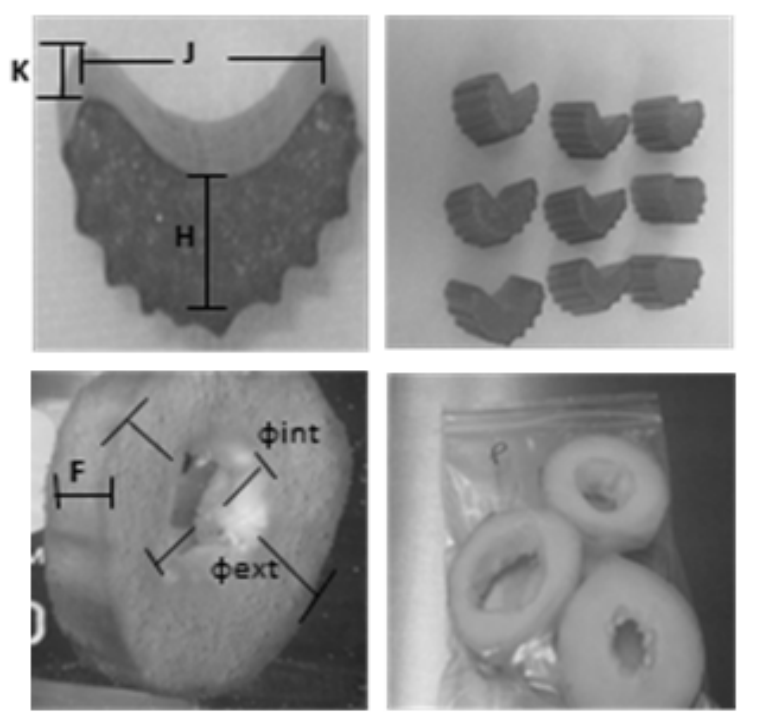

Figura 1. Formas experimentales de pepino y apio para tratamiento IV con solución isotónica.

Análisis fisicoquímicos. Se determinó el contenido de humedad ( $\% \mathrm{p} / \mathrm{p}$ ) a $105^{\circ} \mathrm{C}$ hasta peso constante según la norma AOAC 7.003-84 (21); para el pH, se usó un titulador automático por inmersión del electrodo en la muestra (Hanna Istruments pH 211, Chula Vista, USA), previa calibración con soluciones tampón de $\mathrm{pH}$ 2, 4, 7 y 10 a $25^{\circ} \mathrm{C}$ AOAC 981.12 (22); el contenido de sólidos solubles ('Brix) por lectura refractométrica AOAC 932.12 (22) en un refractómetro (Atago, Tokyo, Japón), a $20^{\circ} \mathrm{C}$; la acidez por titulación con $\mathrm{NaOH} 0.1 \mathrm{~N}$, utilizando fenolftaleína como indicador; el valor de acidez se expresó en gramos de ácido cítrico por $100 \mathrm{~g}$ de muestra según la norma AOAC 942.15 (22); la actividad de agua $\left(a_{w}\right)$ según la norma AOAC 978.19B (23), con un higrómetro de punto de rocío a $25^{\circ} \mathrm{C}$ (Aqualab Decagón modelo CX3 \pm 0.03 , Pullman, WA, USA); la densidad de las disoluciones impregnación ( $\rho$ dis) por el método del picnómetro AOAC 945.06 (23); la densidad aparente $\left(\rho_{\mathrm{apm}}\right)$ por la relación de masa de la muestra y volumen desplazado por la misma en una probeta. 
Análisis microestructural. Se utilizó la técnica de observación por microscopía electrónica de barrido (SEM) (JEOL JSM 5950 LV, Jeol Tokio, Japón), 25 Pa de vacío y $15 \mathrm{kv}$ de corriente eléctrica, para observar los espacios intercelulares del tejido de las hortalizas a impregnar, con el objeto de analizar su potencial porosidad para alojar la solución de impregnación (SI). Una sección transversal y longitudinal de cada hortaliza fue tomada de la zona media de cada muestra y sometida a proceso de liofilización para retirarle el $97 \%$ de contenido de agua. Antes de su observación por SEM, las muestras fueron sometidas a vacío y recubiertas con una fina capa de oro para tener la capacidad de reflejar los electrones que distribuyen la intensidad de las señales en la observación.

Proceso de impregnación al vacio (IV). Los experimentos de IV fueron llevados a cabo en un equipo diseñado en la Universidad Nacional de Colombia Sede Medellín, el cual consiste en una cámara de vacío de acero inoxidable con un sistema electromecánico en su interior para desplazar la muestra dentro y fuera del recipiente que contiene la SI, además un sistema de vibración para eliminar la SI adherida en la superficie de las mismas (Figura 2).
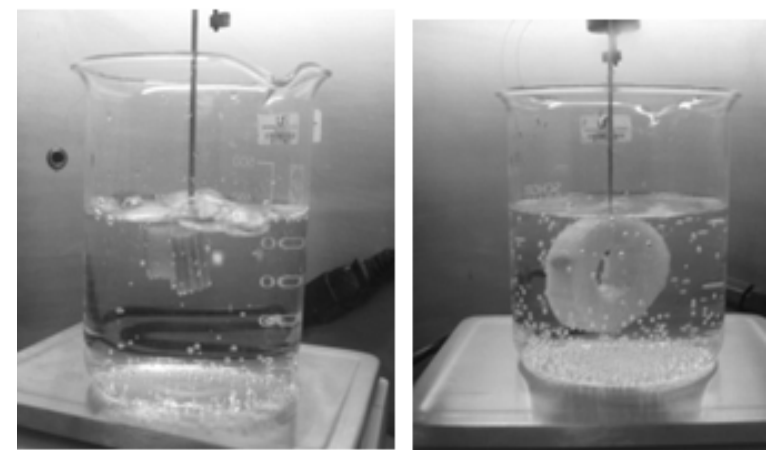

Figura 2. Muestras de apio y pepino durante el proceso IV.

El vacío aplicado fue de $5.9 " \mathrm{Hg}$, verificado en un vacuómetro $(0-30 " \mathrm{Hg})$, el tiempo de cada etapa de proceso fue de 5 minutos, donde se determinó la evolución del peso y volumen de la muestra y de la SI en cada etapa. La respuesta a la IV se expresó en términos de $\mathrm{X} 1, \gamma 1, \mathrm{X}, \gamma \mathrm{y}$
Ee. La relación de los parámetros de IV se ilustra en la ecuación 1 (24), donde $r$ representa la relación de compresión $\left(\mathrm{P}_{2} / \mathrm{P}_{1}\right)$. La metodología y el cálculo de los parámetros de impregnación, ha sido descrito por algunos autores $(18,24)$.

$$
\text { Ee }(\gamma-1)=(X-\gamma) r+\gamma 1
$$

Las SI utilizadas para apio y el pepino fueron de características isotónicas (aw $\mathrm{SI}=\mathrm{aw}$ vegetal), $\mathrm{NaCl}$ al $1.00 \%$ y $1.22 \%$ $\mathrm{p} / \mathrm{p}$ respectivamente.

Análisis de datos. Los resultados fueron analizados a partir de ANOVA, utilizando el método LSD (mínimas diferencias significativas) como método de comparaciones múltiples, con un nivel de confianza del $95 \%(a=0.05)$. El análisis de varianza fue realizado con el paquete estadístico STATGRAPHICS PLUS versión 5.1.

\section{RESULTADOS}

Caracterización fisicoquímica. La tabla 1 presenta los valores medios más las desviaciones estándar de las propiedades fisicoquímicas del apio y pepino frescos.

Tabla 1. Caracterización fisicoquímica de apio y pepino fresco.

\begin{tabular}{lcc}
\hline Característica & Apio & Pepino \\
\hline Humedad $(\%)$ & $94.1 \pm 1.7$ & $96.8 \pm 0.3$ \\
$\mathrm{pH}$ & $5.3 \pm 0.6$ & $5.7 \pm 0.3$ \\
$\circ$ Brix & $4.4 \pm 1.1$ & $3.4 \pm 0.5$ \\
Acidez $(\%)$ & $0.06 \pm 0.02$ & $0.05 \pm 0.02$ \\
$\mathrm{a}_{\mathrm{w}}$ & $0.996 \pm 0.011$ & $0.997 \pm 0.019$ \\
$\rho_{\mathrm{apm}}\left(\mathrm{kg} / \mathrm{m}^{3}\right)$ & $0.951 \pm 0.039$ & $0.962 \pm 0.025$ \\
$\rho_{\mathrm{dis}}\left(\mathrm{kg} / \mathrm{m}^{3}\right)$ & $1.022 \pm 0.006$ & $1.012 \pm 0.001$ \\
\hline
\end{tabular}

Caracterización del proceso de impregnación al vacío. La figura 3 presenta el comportamiento de las variables de respuesta a la IV en las posiciones 1,2 y 3 para los vegetales en estudio.

Caracterización microestructural del apio y pepino. La figura 4, presenta micrografías tomadas por SEM de los 

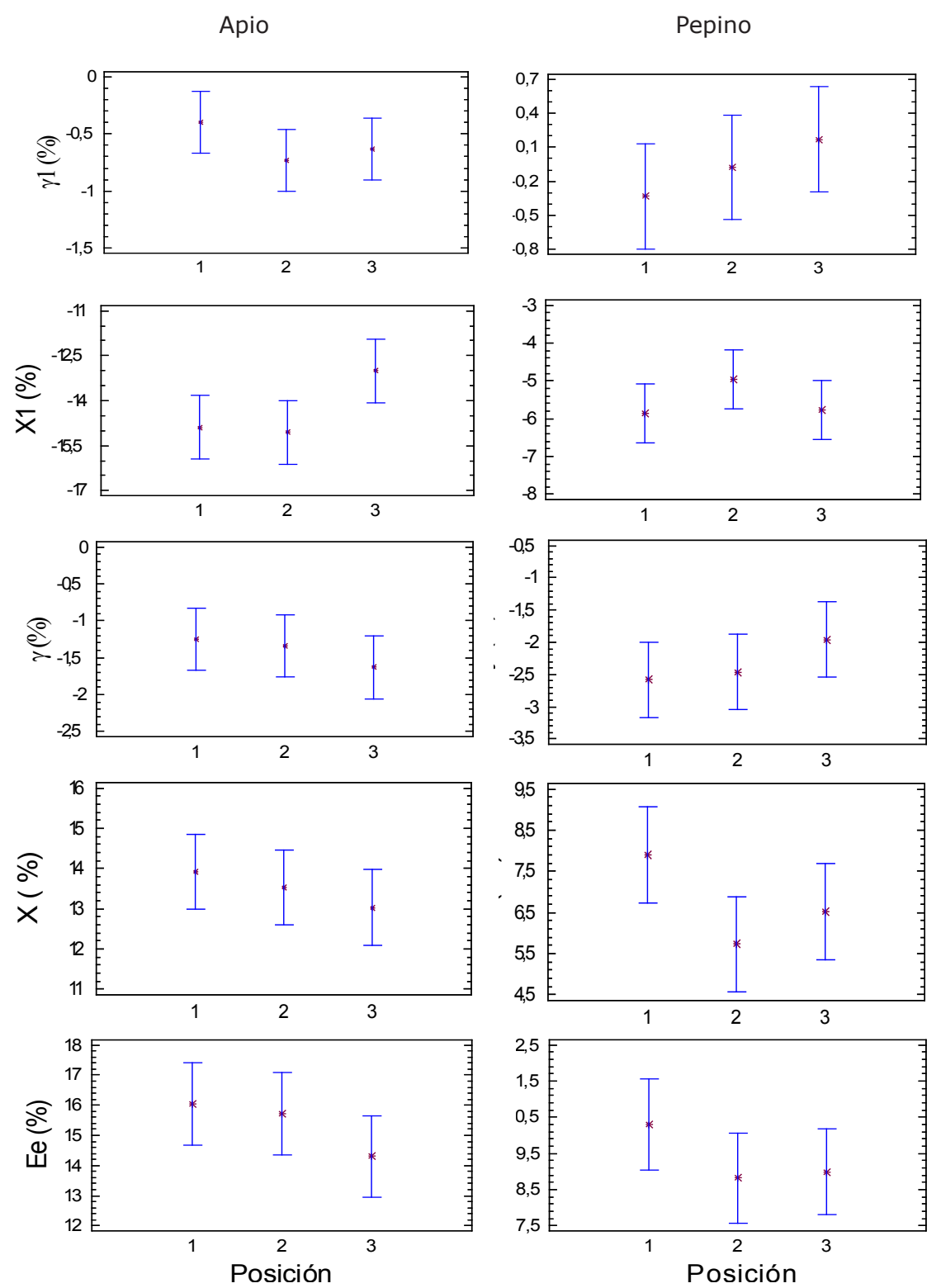

Figura. 3. Valores medios con intervalos LSD (95\%) de los parámetros de impregnación en apio y pepino en función de la posición.

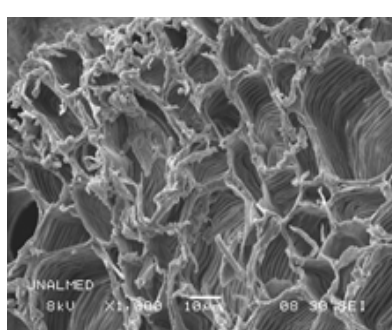

(a) (Apio fresco)

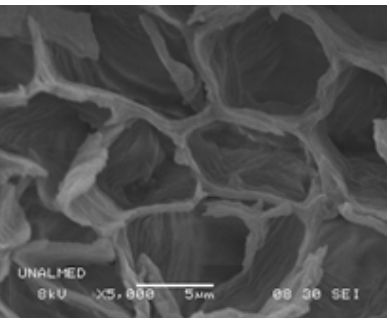

(b) (Apio IV)

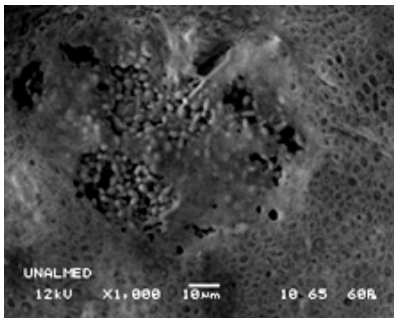

(c) (Pepino fresco)

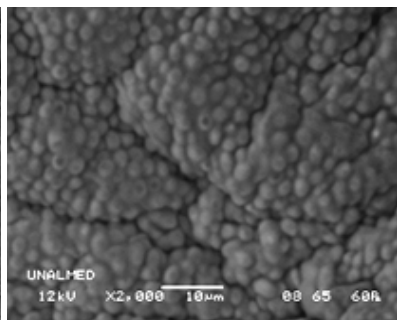

(d) (Pepino IV)

Figura. 4. Imágenes por SEM de los tejidos de apio y pepino en estado fresco (a) y (c) e impregnados (b) y (d) respectivamente. 
tejidos de apio y pepino en estado fresco e impregnados, entre 1000 y 5000 aumentos.

\section{DISCUSIÓN}

Caracterización fisicoquímica. LoS resultados obtenidos para las propiedades fisicoquímicas concuerdan con valores los reportados por otros autores para las dos matrices alimenticias $(16,19,20,25)$, presentando coeficientes de variabilidad aceptables.

\section{Caracterización del proceso de} impregnación al vacío. Para el apio y el pepino, el ANOVA no mostró diferencias significativas $(p>0.05)$ en ninguna de las variables de impregnación con respecto al factor posición. Se observan valores promedios negativos para el parámetro X1 en el apio $(-14.32 \pm 2.75 \%)$ y en el pepino $(-5.51 \pm 1.76 \%)$, lo cual representa una pérdida de líquido nativo desde el interior de la estructura durante la etapa de vacío, atribuido principalmente al tiempo y presión de vacío aplicado, que hace que el alto contenido de humedad en los espacios intercelulares migre hacía el líquido de impregnación. En el apio, la posición 3 presenta una tendencia de menor pérdida de líquido nativo, lo cual podría asociarse a que esta zona es más cercana al punto de disposición final del agua que es conducida hacia las hojas por el sistema vascular del tallo; para el caso del pepino este fenómeno fue más uniforme. La salida de líquido nativo como respuesta al proceso IV en estructuras vegetales, ha sido reportado en zanahoria, berenjena y remolacha (13), en uchuva impregnada con soluciones de microorganismos probióticos (26), en plátano impregnado con soluciones antipardeantes (27), en hongos Pleurotus ostreatus impregnados con soluciones conservantes (28), entre otros.

La deformación volumétrica $\left(\begin{array}{lll}\gamma 1 & \text { y } & \gamma\end{array}\right)$ presentó bajos coeficientes de variabilidad en ambas estructuras, dentro de un rango similar de valores; y no se observaron diferencias significativas con respecto a la posición $(p>0.05)$. La deformación volumétrica se asocia al acoplamiento del MHD y al FDR que producen la contracción en la estructura del tejido vegetal $(3,13$, 12), siendo baja para ambas estructuras. Para el apio se observó una ligera deformación hasta un $1 \%$ en la etapa de vacío $(\gamma 1)$, causado por la salida de líquido nativo que induce a un colapso estructural (contracción volumétrica); mientras que el restablecimiento de la presión atmosférica contribuye a incrementar la deformación volumétrica global $(\gamma)(-1.41 \pm 1.03 \%)$, donde las células alargadas poligonales con paredes de espesor irregular pertenecientes al colénquima de la estructura, a pesar de verse rígidas presentan cierta flexibilidad en los amplios espacios intercelulares (29).

El pepino no presentó cambios volumétricos apreciables en la etapa de vacío $(\gamma 1)$, pero la presión positiva inducida por el líquido de impregnación en la etapa atmosférica, incide sobre la células parenquimatosas densamente empacadas, sufriendo una mayor deformación global $(\gamma)(-2.33 \pm 1.26 \%)$ que el apio, debido a la menor rigidez de sus paredes celulares. Un fenómeno similar ha sido observado en plátano verde (Musa paradisiaca) (27).

Las fracción volumétrica de impregnación $(X)$, muestra al apio como una estructura de mayor capacidad de impregnación $(X=13.49 \pm 2.32 \%)$ debido a la mayor porosidad disponible para el proceso IV (15.35 $\pm 3.40 \%)$. Para el pepino, la estructura a pesar de presentar menores valores de $X(6.72 \pm 2.72 \%)$ y menor porosidad disponible al proceso IV $(9.35 \pm 2.57 \%)$ que el apio, se considera igualmente una estructura adecuada para incorporar a través de la SI, CFA, mejoradores de textura y componentes que modifiquen el perfil sensorial de sabor u otros atributos de calidad.

Caracterización microestructural del apio y pepino. La figura 4(a) presenta las células colenquimáticas del tejido vascular del apio, formando cordones discretos que circunscriben cavidades continuas en formas elípticas por debajo de la epidermis, de tamaño irregular que oscila entre (20 x $40 \mu \mathrm{m})$ y $(10 \times 15 \mu \mathrm{m})$, optimas para alojar 
la SI, en compañía de la fase líquida original presente del tejido fresco. En la figura 4(b) se observa el tejido de apio sometido al proceso IV, donde las cavidades denotan una estructura ligeramente diferente al tejido fresco, debido a una contracción volumétrica de la estructura durante la etapas del proceso, siendo coherente con los valores negativos de $\gamma 1$ y $\gamma$; esta situación resulta como se comentó anteriormente a la salida de líquido nativo en la etapa de vacío y la contracción por efecto de la restauración de la presión atmosférica en la etapa final.

Para el pepino fresco, la figura 4(c), permite apreciar las células del colénquima densamente empacadas debajo del corte transversal de la rodaja, siendo éstas, la estructura sostén que le confiere un carácter elástico y la propiedad de hinchar su pared celular al momento de hidratarse (30). El tejido impregnado, ilustrado en la figura 4(d), permite apreciar las células con un mayor grado de aglomeración, debido a una mayor contracción volumétrica (superior al apio), alcanzada en la etapa final, con valores de $\gamma=-2.33 \pm 1.26 \%$.

En conclusión, la aplicación de la técnica de IV en apio y pepino, permitió identificar estas estructuras como adecuadas para la incorporación de un líquido de impregnación de carácter isotónico al interior del tejido, lo cual es relevante por sus posibles aplicaciones futuras en la incorporación de otros componentes que permitirían mejorar sus atributos de calidad, ampliar su vida útil y además proporcionar un mayor valor agregado, por ejemplo componentes con actividad fisiológica, como vitaminas, minerales, probióticos, prebióticos, entre otros. El proceso IV, confiere cambios microestructurales en los tejidos (contracción volumétrica), presentando una mayor aglomeración de las células colenquimáticas.

\section{REFERENCIAS}

1. Fito P y Chiralt A. Vacuum impregnation of plant tissues. En: S.M. Alzamora, M. S. Tapia, \& A. López-Malo., Design of minimal processing technologies for fruit and vegetables. 1ra Ed. Maryland: Aspen Publishers; 2000. p. 293-305.

2. Fito $P$, Chiralt $A$, Betoret $N$, Grass $M$, Cháfer M, Martinez-Monzo J et al. Vacuum impregnation and osmotic dehydration in matrix engineering application in functional fresh food development. J Food Eng 2001; 49 (23):175-183.

3. Guillemin A, Guillon $F$, Degraeve $P$, Rondeau $C$, Devaux M, Huber $F$ et al. Firming of fruit tissues by vacuuminfusion of pectin methylesterase: Visualization of enzyme action. Food Chem 2008; 109 (2): 368-378.
4. Phoon P, Gomez F, Antonio V, Dejmek P. Pulsed electric field in combination with vacuum impregnation with trehalose improves the freezing tolerance of spinach leaves. J Food Eng 2008, 88 (1):144-148.

5. Alzamora S, Salvatori D, Tapia M, López- Malo A, Welti-chanes J, Fito P. Novel functional foods from vegetable matrices impregnated with biologically active compounds. J Food Eng 2005; 67(1-2):205-214.

6. Betoret $N$, Puente $L$, Díaz $M$, Pag $M$, García M, Gras M et al. Development of probiotic-enriched dried fruits by vacuum impregnation. J Food Eng 2003; 56 (2-3): 273-277.

7. Atarés L, Chiralt A, Gonzalez- Martinez C. Effect of the impregnated solute on air drying and rehydration of apple slices (cv. Granny Smith). J Food Eng 2009; 91 (2): 305-310. 
8. Restrepo A, Cortés M, Rojano B. Determinación de la vida útil de fresa (Fragaria ananassa Duch.) fortificada con vitamina E. DYNA 2009; 159 (76): 163-175.

9. Botero A. Aplicación de la ingeniería de matrices en el desarrollo de uchuva (Physalis peruviana L.) mínimamente procesada fortificada con calcio y vitaminas C y E. [Tesis de Maestría]. Colombia: Universidad de Antioquia, Facultad de Química Farmacéutica; 2008.

10. Anino S, Salvatori D, Alzamora S. Changes in calcium level and mechanical properties of apple tissue due to impregnation with calcium salts. Food Res Int 2006; 39 (2): 154164.

11. Castello M, Fito $P$, Chiralt A. Effect of osmotic dehydration and vacuum impregnation on respiration rate of cut strawberries. LWT 2006; 39(10):1171-1179.

12. Cortés M. Desarrollo de productos de manzana deshidratados enriquecidos con vitamina E. [Tesis de Doctorado]. España: Universidad Politécnica de Valencia, Departamento de Tecnología de Alimentos; 2004.

13. Grass $M$, Vidal-Brotons $D$, Betoret $\mathrm{N}$, Chiralt A, Fito P. The response of some vegetables to vacuum impregnation. Innov Food Sci Emerg 2002; 3(3):263-269.

14. Grass. M.; Vidal-Brotons, D.; Betoret, N.; Chiralt, A.; Fito, P. (2003). Calcium Fortification of vegetables by vacuum impregnation Interactions with cellular matrix. J Food Eng 56(1):279-284.

15. Karakas B, Yildiz F. Peroxidation of membrane lipids in minimally processed cucumbers packaged under modified atmospheres. Food Chem 2007; 100(3): 1011-1018.
16. Gomez P, Artés F. Controlled atmospheres enhance postharvest green celery quality. Postharv Biol Technol 2004; 34(2):203-209.

17. Zhaoa Y, Xieb J. Practical applications of vacuum impregnation in fruit and vegetable processing. Food Sci \& Tech 2004; 15(9):434-451.

18. Salvatori $D$, Andrés $A$, Chiralt $A$, Fito $P$. The response of some properties of fruits to vacuum impregnation. J Food Eng 1998; 21(1):59-73

19. Rizzo V, Muratore G. Effects of packaging on shelf life of fresh celery. J Food Eng 2009; 90(1):124-128.

20. Dermesonlouoglou E.K, Pourgouri S, Taoukis P.S. Kinetic study of the effect of the osmotic dehydration pretreatment to the shelf life of frozen cucumber. Inn Food Sci Em Technol 2008; 9(4):542-549.

21. AOAC International. Official Methods of Analysis of Association of Official Agricultural Chemists (AOAC) International. 17th edition. Maryland, USA: Horwitz, W (Ed); 2000.

22. AOAC International. Official Methods of Analysis of Association of Official Agricultural Chemists (AOAC) International. 15th edition. Virginia, USA: Helrich, K(Ed); 1990.

23. AOAC International. Official Methods of Analysis of Association of Official Agricultural Chemists (AOAC) International. 16th edition. Virginia, USA: Helrich, K(Ed); 1998.

24. Martínez- Navarrete $N$, Andrés $A$, Chiralt A, Fito P. Termodinámica y Cinética de Sistemas Alimento y Entorno. En: Martínez-Navarrete N. Mecanismo difusional en Alimentos. España: Servicio de publicaciones Universidad Politécnica de Valencia; 1998. 
25. Raffo A, Sinesio F, Moneta E, Nardo $N$, Peparaio $M$, Paoletti $F$. Internal quality of fresh and cold stored celery petioles described by sensory profile, chemical and instrumental measurements. Eur Food Res Technol 2006; 222(5-5):590-599.

26. Marín Z. Viabilidad de desarrollo de uchuva (Physalis peruviana L.) mínimamente procesada enriquecida con microorganismos probióticos a partir de la ingeniería de matrices. [Tesis de Maestría]. Colombia: Universidad Nacional de Colombia, Departamento Ingeniería Agrícola y Alimentos; 2009.

27. Dávila R. Aplicación de la ingeniería de matrices en el desarrollo de plátano (Musa paradisiaca) verde mínimamente procesado. [Tesis de Maestría]. Colombia: Universidad Nacional de Colombia, Departamento Ingeniería Agrícola y Alimentos; 2009.
28. Ruiz, M. Efecto de dos atmosferas de empaque en hongos comestibles (Pleorotus ostreatus) tratados mediante impregnación a vacío con una solución conservante. Vitae 2010; 17(1):11-19.

29. Eichhorn S, Evert R, Hamilton P. Biología de las plantas. 1ra edición. México: Editorial Reverte S.A.; 1992.

30. Valla J: Botánica Morfología de las plantas superiores. 1a ed. Buenos Aires: Editorial Hemisferio Sur; 2007. 